\title{
Transcription factor Runx2 is a regulator of epithelial-mesenchymal transition and invasion in thyroid carcinomas
}

\author{
Dong-Feng Niu*, Tetsuo Kondo*, Tadao Nakazawa, Naoki Oishi, Tomonori Kawasaki, Kunio Mochizuki, \\ Tetsu Yamane and Ryohei Katoh
}

Runx2/Cbfa1 is a member of the Runt-related transcription factor family and is an essential regulator of osteoblast/ chondrocyte differentiation. Recently, aberrant expression of Runx2 and its oncogenic functions have been identified in the progression and metastasis of human cancers. In this study, we investigated the expression profile of Runx family genes in normal thyroid tissue, non-neoplastic but abnormal thyroid tissue, various types of thyroid tumors and representative human thyroid carcinoma cell lines. Using reverse transcriptase-PCR and western blotting, we found that Runx2 was consistently upregulated in papillary carcinomas (PCs) and thyroid carcinoma cell lines compared with normal thyroid tissue. With immunohistochemistry, we observed negative or focal immunoreactivity of Runx2 in the nuclei of normal thyroid follicular cells. None of the non-neoplastic thyroid tissues, including Graves' thyroid and adenomatous goiter, had diffuse positivity of Runx2. Expression of Runx2 in benign follicular adenomas varied from negative to diffusely positive. Meanwhile, all malignant thyroid tumors showed some Runx2 immunopositivity. It was diffuse and intense in $83 \%(19 / 23)$ of PCs, $71 \%$ (5/7) of follicular carcinomas (FCs) and 40\% (4/10) of undifferentiated carcinomas (UCs). In thyroid carcinoma cell lines, the MEK inhibitor U0126 suppressed Runx2, suggesting an association of the MAPK/ERK pathway with Runx2 regulation. Effective silencing of Runx2 by short interfering RNA (siRNA) demonstrated downregulation of EMT-related molecules (SNAI2, SNAI3 and TWIST1), MMP2 and vasculogenic factors (VEGFA and VEGFC) in thyroid carcinoma cells. We also confirmed that Runx2 silencing suppresses thyroid carcinoma cell invasion in transwell assays. In conclusion, this study provides insight into the potential molecular mechanism of thyroid cancer invasion. Our data suggest that enhanced Runx2 is functionally linked to tumor invasion and metastasis of thyroid carcinoma by regulating EMT-related molecules, matrix metalloproteinases and angiogenic/lymphangiogenic factors.

Laboratory Investigation (2012) 92, 1181-1190; doi:10.1038/labinvest.2012.84; published online 28 May 2012

KEYWORDS: epithelial-mesenchymal transition; immunohistochemistry; real-time PCR; Runx2; thyroid carcinoma

Follicular cell-derived thyroid cancer is the most common malignancy of the endocrine organs, and its incidence rate has steadily increased over the last decade. More than $95 \%$ of thyroid carcinomas are derived from follicular cells encompassing a spectrum of differentiation from comparatively indolent carcinomas, including follicular carcinoma (FC) and papillary carcinoma (PC), to the most invasive and lethal human malignancy, undifferentiated carcinoma (UC). ${ }^{1}$ This wide spectrum of progression has been closely linked with a pattern of cumulative genetic defects and epigenetic gene dysregulation that correlates with tumor differentiation, metastatic potential and aggressiveness. ${ }^{2}$ However, the molecular mechanism of invasion and the epithelial-mesenchymal transition (EMT) phenomenon in thyroid carcinoma must still be clarified.

The mammalian runt-related transcription factor (Runx) genes, including RUNX1 (AML1/CBFA2), RUNX2 (AML2/ CBFA1) and RUNX3 (AML3/CBFA3), encode transcription factors that are master regulators of proliferation and differentiation during embryonic development. Runx proteins form a heterodimeric complex with a non-DNA-binding partner, that is, a core-binding factor- $\beta(\mathrm{CBF} \beta)$. All three Runx proteins have a conserved 128-amino acid Runt domain that mediates DNA binding and heterodimerization 
with the $\mathrm{CBF} \beta$. Despite their highly homologous Runt domains, Runx family genes show tissue-specific divergent functions. For instance, Runx1 is essential for definitive hematopoiesis including erythroid cells, granulocytes, macrophages and lymphocytes. Runx 2 is required for chondroosteoblast lineage differentiation. Runx3 is associated with the regulation of gastric epithelial growth, thymopoiesis and neurogenesis of the dorsal root ganglia. ${ }^{3}$

Aberrant regulation of the Runx family has been highlighted recently in tumor pathogenesis. Runx1 is frequently involved in acute myelogenous leukemias and myelodysplastic syndromes through acquired chromosomal rearrangement and point mutations. ${ }^{4}$ Overexpression of Runx2 is observed in prostate, pancreas and breast cancers. ${ }^{5,6}$ Functional inactivation of Runx3 by hemizygous deletion, promoter DNA hypermethylation and/or histone modification were found in human solid tumors. ${ }^{7}$

Although tumor-suppressive roles have been suggested in Runx 1 and Runx3, Runx 2 is being recognized as a tumorpromoting factor in several human cancers. Runx 2 promotes the metastatic ability of cancer cells by enhancing the secretion of various types of matrix metalloproteinases (MMPs) and VEGF, a key angiogenic factor. ${ }^{8-10}$ Furthermore, upregulation of Runx2 induces EMT-related molecules such as SOX9, Snail2/Slug/SNAI2 and SMAD3 that potentially enhance invasion of cancer cells. ${ }^{11,12}$ In thyroid cancers, overexpression of EMT-related proteins, including Runx2, has been reported; however, little is known about the expression profile of the Runx family and their biological functions in thyroid carcinogenesis. ${ }^{13}$

In this study, we hypothesized that the regulation of cancer invasion and the EMT phenomenon in thyroid carcinomas could be attributed to functional aberrations of the Runx family. Here, we evaluate the expression profile of Runx genes in various thyroid tissues and thyroid carcinoma cell lines. Following confirmation of Runx2 upregulation, we explore the cancer-promoting properties of Runx2 in thyroid carcinomas.

\section{MATERIALS AND METHODS Case Selection}

We investigated 107 surgical specimens representing a range of thyroid pathologies from routine surgical pathology files at University of Yamanashi Hospital, Yamanashi, Japan, including normal thyroid (21 cases), Graves' disease (11 cases), adenomatous goiter (16 cases), follicular adenoma (19 cases), FC ( 7 cases), PC ( 23 cases) and UC (10 cases). All slide specimens were stained with hematoxylin-eosin (HE), and the diagnosis was made on the basis of the World Health Organization classification. ${ }^{14}$ Normal thyroid tissues were obtained from patients who underwent subtotal or total thyroidectomy for PC. The snap-frozen tissues were stored at $-80{ }^{\circ} \mathrm{C}$ before subsequent isolation of their RNA. The institutional ethics board of the University of Yamanashi approved the protocols.

\section{Cell Lines and Cell Culture}

Human thyroid carcinoma-derived cell lines, WRO, TPC-1, KTC-1, 8505C, 8305C and TT, have already been described. ${ }^{15,16}$ UA-1 and UA-2 were originally established cell lines derived from human UC. Cells were maintained in RPMI-1640 (GIBCO, Grand Island, NY, USA) supplemented with $10 \%$ fetal bovine serum (FBS), streptomycin sulfate $(100 \mu \mathrm{g} / \mathrm{ml})$ and penicillin $\mathrm{G}$ sodium $(100 \mathrm{U} / \mathrm{ml})$. Cells were cultured in a standard humidified incubator at $37^{\circ} \mathrm{C}$ in a $5 \%$ carbon dioxide atmosphere.

To determine RUNX2 expression, cultured cells were treated with graded concentrations of FBS $(0,1,5$ and 10\%) in medium for $24 \mathrm{~h}$ after $24 \mathrm{~h}$ of serum starvation. For MEK inhibition, U0126 (Calbiochem, San Diego, CA, USA) was added to the medium at a concentration of $4 \mu \mathrm{M}$ for $1 \mathrm{~h}$, followed by $10 \%$ FBS treatment. To prepare the cell pellets, semiconfluent plates were washed, gently scraped and centrifuged into pellets that were coated in $2 \%$ bactoagar until solidified, fixed in $10 \%$ formalin and embedded in paraffin.

\section{RNA Extraction and Reverse Transcription-PCR}

We isolated total RNA from fresh frozen tissues and cultured cells using TRIzol (Invitrogen, Carlsbad, CA, USA) and generated cDNA using the TaqMan reverse transcription (RT) reagent kit (Applied Biosystems, Foster City, CA, USA). Specific PCR primers targeted for RUNX1, RUNX2 and RUNX3 and glyceraldehyde-3-phosphate dehydrogenase $(G A P D H$, as an internal control) were designed as listed in Table 1. We used the HotStarTaq DNA polymerase kit (Qiagen, Tokyo, Japan) for amplification. PCR conditions were as follows: (1) $95^{\circ} \mathrm{C}$ for $15 \mathrm{~min}$; (2) 30 cycles of $94^{\circ} \mathrm{C}$ for $30 \mathrm{~s}, 58^{\circ} \mathrm{C}$ for $30 \mathrm{~s}$ and $72{ }^{\circ} \mathrm{C}$ for $1 \mathrm{~min}$; (3) $72{ }^{\circ} \mathrm{C}$ for $10 \mathrm{~min}$; and (4) $4{ }^{\circ} \mathrm{C}$ hold. Negative controls omitting RT or without cDNA and appropriate positive controls were included in each PCR reaction.

\section{Quantitative Real-Time PCR}

We performed real-time PCR with MyiQ Single-Color Realtime PCR detection system (Applied Biosystems). Approximately $2.5 \mu \mathrm{l}$ cDNA was amplified in each $25 \mu \mathrm{l}$ PCR reaction mix containing $12.5 \mu \mathrm{l}$ of $2 \times$ SYBR Green Master Mix (Qiagen), $0.75 \mu \mathrm{l}$ each of $10 \mathrm{mM}$ forward and reverse primers and $8.5 \mu \mathrm{l}$ of DEPC-treated water. Amplification was started at $95^{\circ} \mathrm{C}$ for $15 \mathrm{~min}$ as the first step, followed by 45 cycles of PCR: at $95^{\circ} \mathrm{C}$ for $30 \mathrm{~s}$, at $58^{\circ} \mathrm{C}$ for $30 \mathrm{~s}$ and $72^{\circ} \mathrm{C}$ for $60 \mathrm{~s}$, successively. We normalized the amount of the target gene mRNA to that of GAPDH using the threshold cycle method, as described in the manufacturer's protocol. PCR primers used in real-time PCR are listed in Table 1.

\section{Immunohistocytochemistry}

We performed immunohistochemical and immunocytochemical analysis on $3 \mu \mathrm{m}$ sections of routinely processed formalin-fixed and paraffin-embedded tissues or the cell pellets described above. A detailed protocol has been 
Table 1 PCR primers

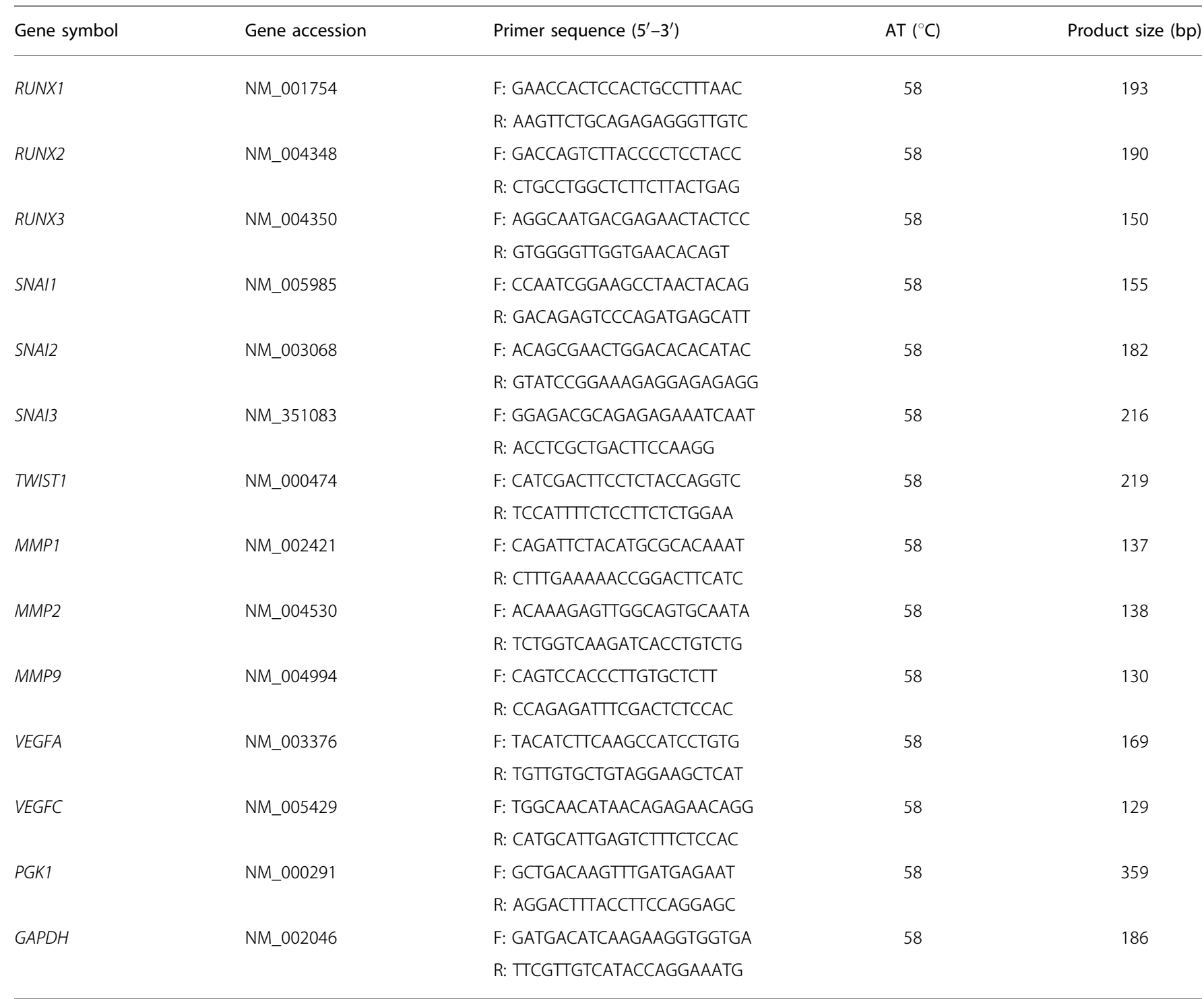

$F$, forward primer; $R$, reverse primer; bp, base pair.

previously described. ${ }^{17}$ Briefly, following antigen retrieval (citrate buffer, $\mathrm{pH}$ 6.0, autoclave for $10 \mathrm{~min}$ ), deparaffinized sections were incubated with anti-Runx-1 mouse-monoclonal antibody (clone 2C10; Abnova, Taipei, Taiwan; 1:50), antiRunx2 mouse-monoclonal antibody (clone 3F5; Abnova; 1:50) or anti-Runx-3 purified MaxPab mouse polyclonal antibody (Abnova; 1:200) for $1 \mathrm{~h}$ at room temperature. To visualize the reaction, we carried out the labeled polymer method (Envision Detection System; Dako, Glostrup, Denmark) according to the manufacturer's instructions. Positive control was as follows: stomach for Runx1, human placenta for Runx2 and endometrium for Runx3. The immunoreactivity was evaluated as absent, mild, moderate and strong: absent, no immunoreactivity; mild, focal immunoreactivity (1 to $9 \%$ positive cells); moderate, intermediate immunoreactivity between mild and strong; strong, diffuse immunoreactivity ( $>50 \%$ positive cells).

Using a protocol of immunocytofluorescence as previously described, ${ }^{18}$ we used primary antibodies and working dilution rates as follows: anti-Runx2 mouse-monoclonal antibody (Abnova; 1:100), anti-SNAI-2/Slug rabbit polyclonal antibody (LS-B554; LifeSpan Biosciences, Seattle, WA, USA; 1:400), anti-MMP-2 rabbit polyclonal antibody (ab37150; Abcam, Cambridge, UK; 1:250) and anti-VEGF-C rabbit polyclonal antibody (no. 18415; Immuno-Biological Laboratories, Gunma, Japan; 1:20). Fluorescein isothiocyanate (FITC)-conjugated immunoglobulin (Dako) was used as a secondary antibody. For a nuclear counterstain, slides were mounted with mounting medium containing $4^{\prime}, 6-$ diamino-2-phenylindole (DAPI/H-1200, Vector Laboratories, 
Burlingame, CA, USA). To visualize the fluorescence, we used an epi-illumination fluorescence light microscope (BX50; Olympus, Tokyo, Japan) and a high sensitivity cooled CCD camera (DP30BW; Olympus).

\section{Protein Isolation and Western Blotting}

Cultured cells were lysed in a radioimmunoprecipitation assay (RIPA) lysis buffer with proteinase inhibitors $(1 \times$ phosphate-buffered saline (PBS), $1 \%$ Nonidet P40, $0.5 \%$ sodium deoxycholate, $0.1 \%$ sodium dodecyl sulfate (SDS), $1 \mathrm{mM}$ phenylmethylsulfonyl fluoride (PMSF), $12 \mu \mathrm{g} / \mathrm{ml}$ aprotinin and $1 \mathrm{mM}$ sodium orthovanadate). Equal amounts of protein $(30 \mu \mathrm{g})$ solubilized in sample buffer were separated on $10 \%$ SDS polyacrylamide gels and transferred electrophoretically to polyvinylidene difluoride (PVDF) membranes. Membranes were blocked in Tris-buffered saline (TBS) containing $0.05 \%$ Tween- 20 plus $5 \%$ nonfat dried milk for $1 \mathrm{~h}$ at room temperature and then probed with primary antibodies at $4{ }^{\circ} \mathrm{C}$ overnight.

Primary antibodies were used at the specified dilutions: anti-Runx2 (1:1000), anti-Snail2/Slug(1:1000), anti-MMP-2 (1:1000), anti-phospho-ERK (Sigma; 1:1000), anti-total ERK (Sigma; $1: 5000)$ and anti- $\beta$-actin (Abcam; $1: 5000)$. Membranes were washed three times for $15 \mathrm{~min}$ each in TBS containing $0.05 \%$ Tween-20 and incubated with horseradish peroxidase (HRP)-conjugated goat anti-mouse/rabbit secondary antibody at 1:2000 dilution (Santa Cruz Biotechnology, Santa Cruz, CA, USA) for $1 \mathrm{~h}$ at room temperature. We visualized the targeted proteins using an enhanced chemiluminescence detection system (Amersham, Buckinghamshire, UK).

\section{Small Interfering RNA Transfection}

We grew WRO and 8305C cells in RPMI-1640 supplemented with $0.5 \%$ FBS in six-well culture plates until $50 \%$ confluence. Runx2 short interfering RNA (siRNA) transfection was performed using HiperFect transfection reagent (Qiagen) according to the manufacturer's instructions. The Runx2 target sequence was $5^{\prime}$-CAGAAGCTTGACTCTAAA-3', and AllStars negative control siRNA (Qiagen) was used as a control. We confirmed alterations of Runx2 expression with RT-PCR, real-time PCR and immunocytochemistry.

\section{Invasion and Migration Assays}

We assessed cell motility in a transwell assay using 24-well plates with uncoated inserts to examine migration or in Matrigel-coated inserts to assess invasiveness (BD Biosciences, Franklin Lakes, NJ, USA). Briefly, the upper and lower culture compartments were separated by polycarbonate filters with a pore size of $8 \mu \mathrm{m}$. After trypsinization, $2 \times 10^{4}$ cells were plated in each insert with $500 \mu \mathrm{l}$ of serum-free medium. The growth medium containing 10\% FBS was used as a chemoattractant in the bottom well. After $24 \mathrm{~h}$ of incubation, cells on the upper surface were removed by scrubbing with a cotton swab. Cells on the lower surface of the membrane were stained with Diff-Quik stain and quantified by light microscopy. Assays were done in triplicate. Data are presented as mean \pm s.d.

\section{Statistics}

We set statistical significance at $P<0.05$, and performed data analyses with SPSS version 13.0 for windows (SPSS, Tokyo, Japan).

\section{RESULTS}

\section{Expression Profiles of Runx Genes in Thyroid Tumors}

We investigated the expression pattern of the Runx gene family in thyroid carcinomas by RT-PCR. The PC tissues had a consistently higher expression level of RUNX1 and RUNX2 mRNA compared with normal thyroid tissues in 4 of 4 cases (Figure 1a). On the other hand, 2 of 4 PC cases had a lower expression level of RUNX3 mRNA.

Then, we focused on Runx 2 in thyroid tumors. Using immunohistochemistry, we assessed the expression of Runx2 protein in various histological types of thyroid (Figure 1b). Table 2 summarizes these immunohistochemical results. We saw positive immunostaining of Runx2 in the nuclei of cells. In normal thyroid tissue, follicular cells exhibited negative (absent) or focal positivity (mild) in 71\% (15/21) and 29\% (6/21) of cases, respectively. In detail, positive follicular cells were cuboidal comprising small follicles. The expression pattern of Runx2 varied from absent to moderate in Graves' thyroid and adenomatous goiter, and we did not see any strong positivity of Runx2 in these non-neoplastic thyroid diseases. Runx2 immunostaining in benign follicular adenomas varied from absent to $11 \%(2 / 19)$ being strong positive. Immunohistochemical intensity of Runx 2 in follicular adenomas was rather weak compared with the intensity in thyroid carcinomas. Furthermore, all malignant thyroid tumors were immunopositive for Runx2. It was diffuse and intense (strong) in 83\% (19/23) of PCs, 71\% (5/7) of FCs and $40 \%$ (4/10) of UCs. Representative immunostaining for Runx 1 and Runx 3 are demonstrated in Figure $1 \mathrm{c}$ and $\mathrm{d}$.

\section{Runx Genes in Thyroid Carcinoma Cell Lines}

We also examined expression of the Runx family in eight thyroid carcinoma cell lines (Figure 2a). Using RT-PCR, we detected RUNX1 mRNA in seven cell lines and RUNX2 mRNA in six cell lines. RUNX3 mRNA was detected in one cell line (8505C cells). All cell lines that expressed Runx family genes were follicular cell derived. We did not detect any Runx family genes in the C cell-derived TT cell line. Western blotting confirmed Runx2 protein expression in KTC-1, TPC-1, WRO, UA-2 and 8305C cells (Figure 2b). Immunocytochemistry demonstrated nuclear localization of Runx2 in WRO, TPC-1 and 8305C cells (Figure 2c). Neither RUNX2 mRNA nor Runx2 protein was detected in $8505 \mathrm{C}$ cells. 
a
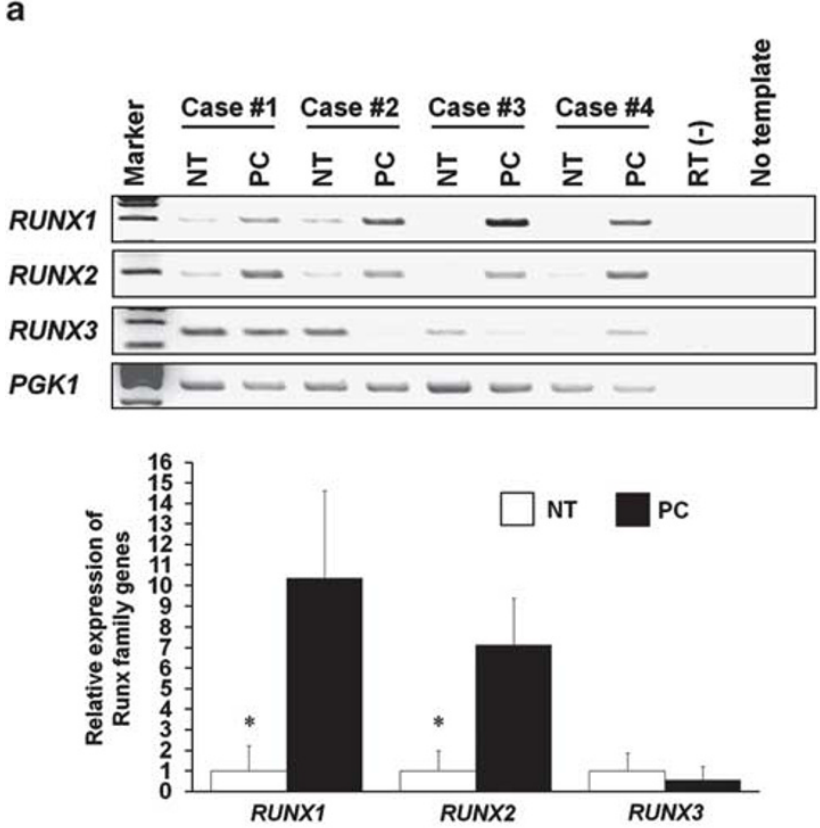

C

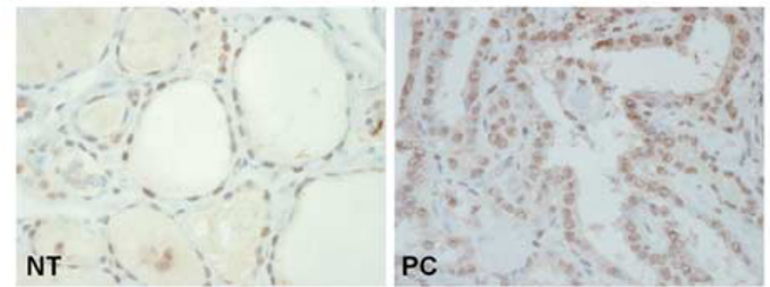

b

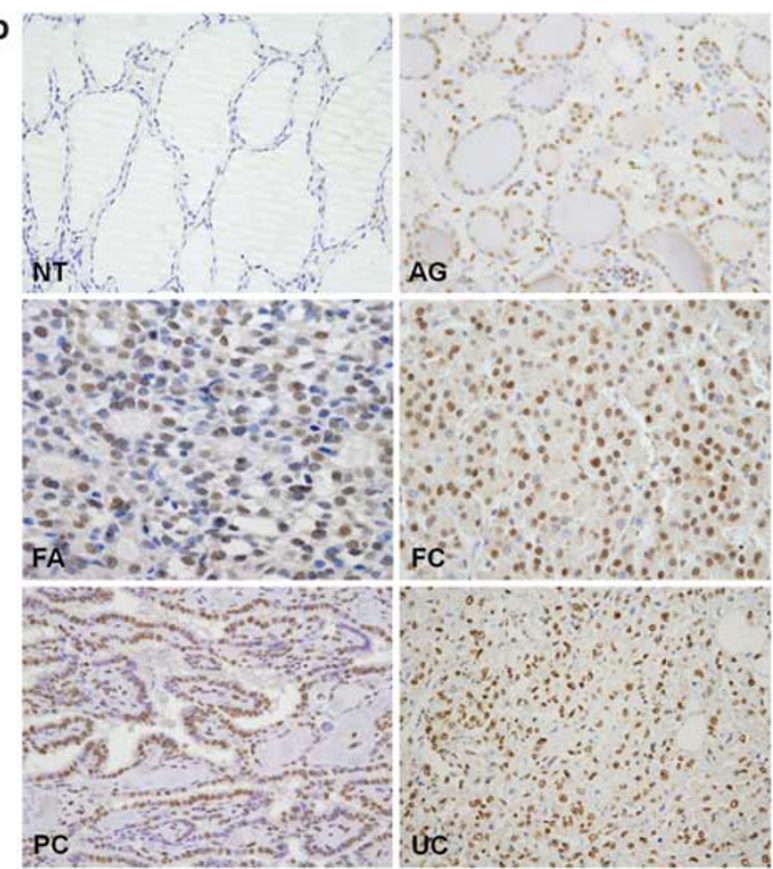

d

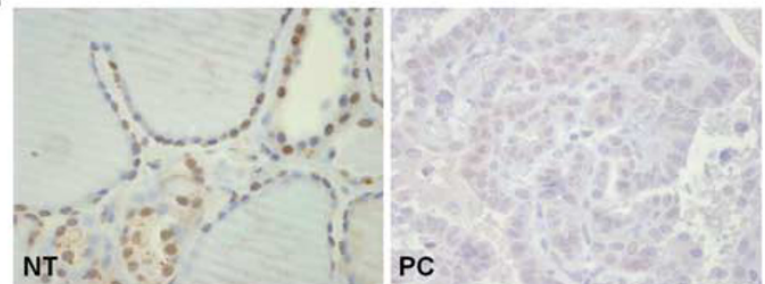

Figure 1 Expression profiles of Runx family genes. (a) RT-PCR shows that the expression level of RUNX1 and RUNX2 mRNAs are significantly higher in PC tissues than in normal thyroid tissues as shown by the densitometric assessments below $\left({ }^{\star} P<0.05\right)$. Although two of four PC cases have a lower expression of RUNX3 mRNA, it is not statistically significant. (b) Immunohistochemistry for Runx2 in thyroid tissues. Follicular cells in normal thyroid (NT) are negative for Runx2. Moderate positivity is observed in adenomatous goiter (AG) and follicular adenoma (FA). A diffuse positivity pattern is exhibited in malignant thyroid tissue including follicular carcinoma (FC), papillary carcinoma (PC) and undifferentiated carcinoma (UC). (c) Immunohistochemistry for Runx1. Follicular cells in NT show moderate positivity for Runx1. Diffuse immunopositivity of Runx1 is observed in PC. (d) Immunohistochemistry for Runx3. Normal follicular cells are moderate positive for Runx3. Tumor cells of PC show mild positivity for Runx3.

\section{Regulation of Runx2 Expression in Thyroid Carcinoma Cells}

Based on enhanced expression of Runx2 in thyroid carcinomas, we investigated the relationship between mitogen-activated protein kinase/extracellular signal-regulated kinase (MAPK/ERK) signaling consequence and Runx2 expression. We stimulated Runx2-positive cells (WRO and TPC-1) with various concentrations of FBS $(0,1,5$ and $10 \%)$ and MEK inhibitor (U0126), and then assessed RUNX2 mRNA expression by RT-PCR (Figure 3a) and real-time PCR (Figure $3 \mathrm{~b}$ ). We found that RUNX2 mRNA increased with serum stimulation in a concentration-dependent manner, whereas treatment of MEK inhibitor significantly suppressed RUNX2 expression in both cell lines $(P<0.05)$. Immunocytofluorescence confirmed induction of Runx2 expression under serum stimulation in WRO and TPC-1 cells (Figure 3c). We further validated the results of PCR,
Table 2 Runx2 protein expression in various thyroid lesions

\begin{tabular}{lrrrrr} 
Histological type & $N$ & \multicolumn{5}{c}{$\begin{array}{c}\text { Immunopositivity } \\
\text { for Runx2 }\end{array}$} \\
\cline { 3 - 6 } & & Absent & Mild & Moderate & Strong \\
\hline Normal thyroid & 21 & 15 & 6 & 0 & 0 \\
Graves' thyroid & 11 & 5 & 4 & 2 & 0 \\
Nodular hyperplasia & 16 & 3 & 11 & 2 & 0 \\
Follicular adenoma & 19 & 5 & 8 & 4 & 2 \\
Papillary carcinoma & 23 & 0 & 0 & 4 & 19 \\
Follicular carcinoma & 7 & 0 & 0 & 2 & 5 \\
Undifferentiated carcinoma & 10 & 0 & 1 & 5 & 4
\end{tabular}

Absent, negative; mild, focal ( 1 to $9 \%$ of cells); moderate, intermediate (10 to $50 \%$ ); strong, diffuse (more than $50 \%$ ). 
a

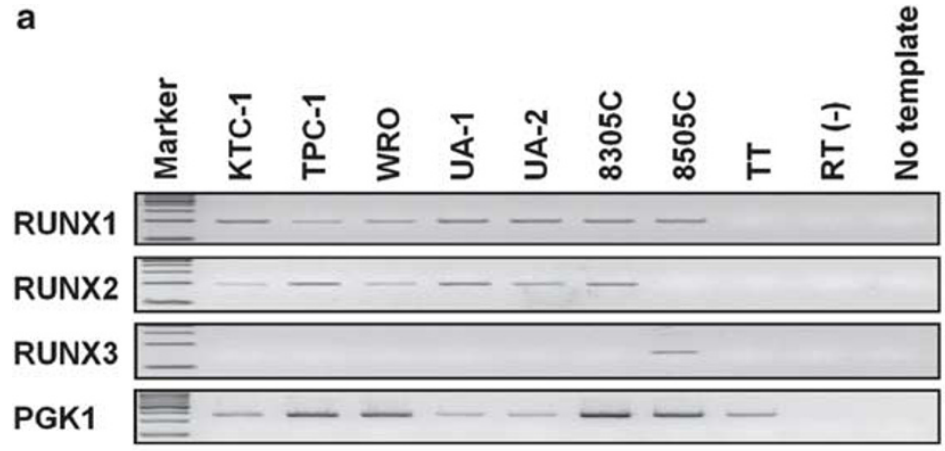

b

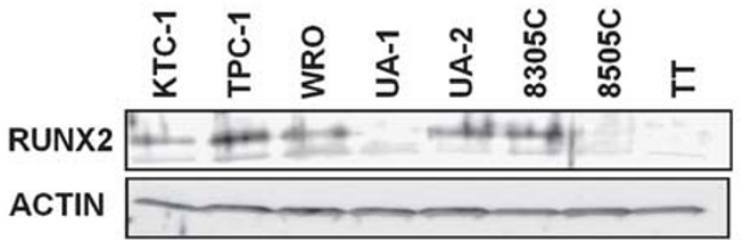

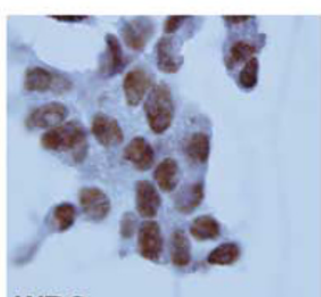

\section{WRO}

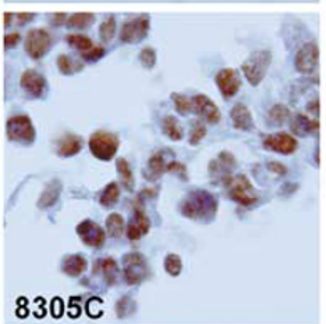

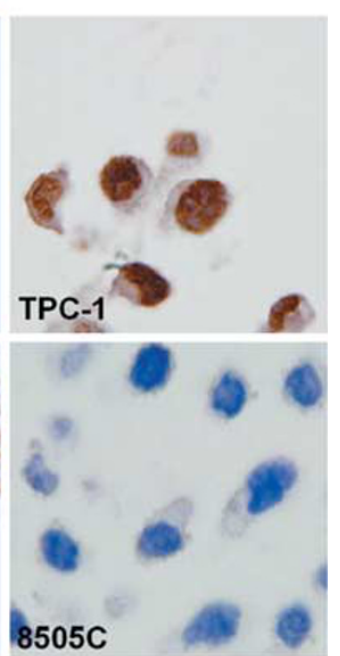

Figure 2 Expression of Runx genes in thyroid carcinoma cell lines. (a) RT-PCR detects RUNX1 mRNA in seven cell lines (KTC-1, TPC-1, WRO, UA-1, UA-2, 8305C and 8505C cells) and RUNX2 mRNA in six cell lines (KTC-1, TPC-1, WRO, UA-1, UA-2 and 8305C cells). RUNX3 mRNA is detected only in 8505 C cells. None of the Runx genes are detected in C cell-derived TT cells. (b) Western blotting shows Runx2 protein expression in KTC-1, TPC-1, WRO, UA-2 and $8305 \mathrm{C}$ cells. (c) Immunocytochemistry demonstrates nuclear localization of Runx2 in WRO, TPC-1 and $8305 \mathrm{C}$ cells. No immunopositivity is detected in 8505 C cells.

a

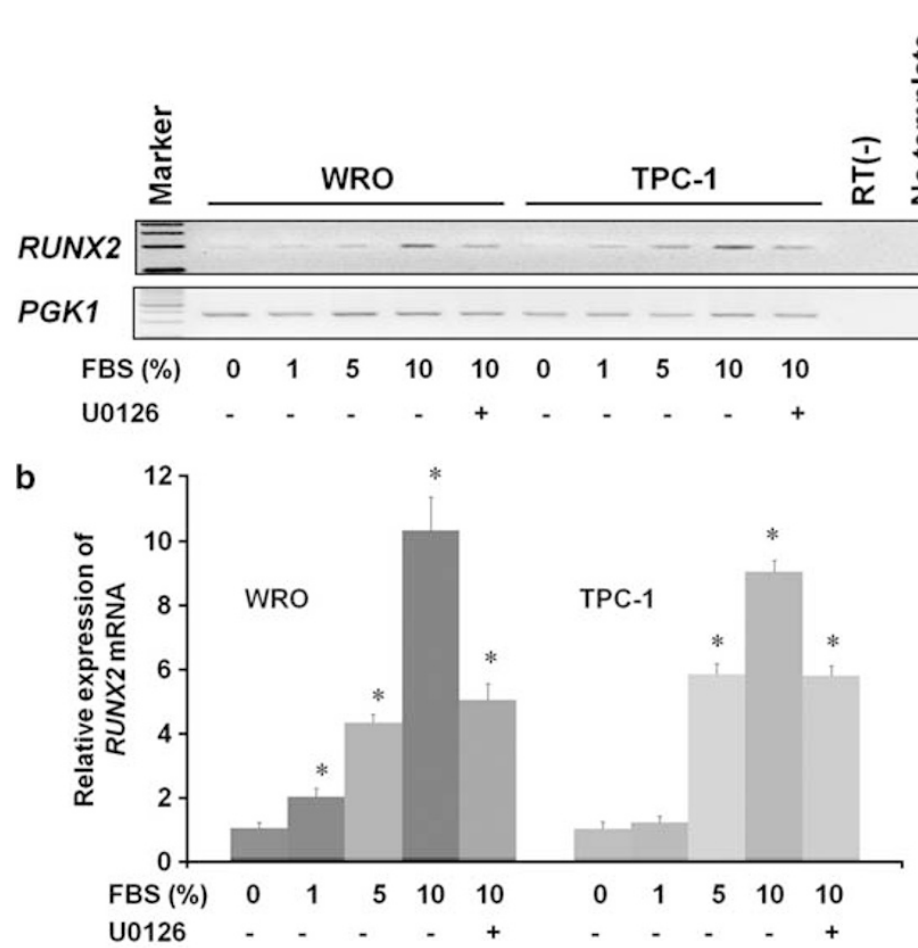

C
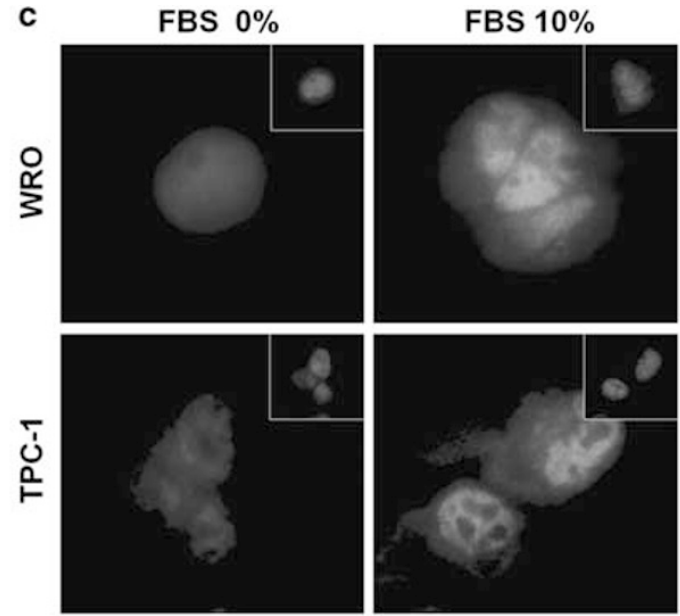

d

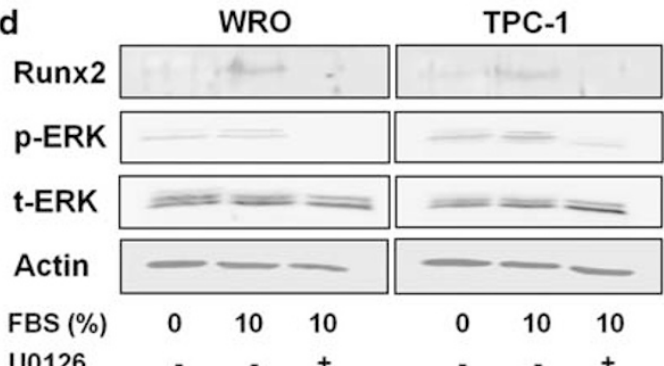

Figure 3 Regulation of Runx2 expression. (a) RT-PCR. Fetal bovine serum (FBS) stimulation increases RUNX2 mRNA in a concentration-dependent manner in WRO and TPC-1 cells, and MEK inhibitor (U0126) significantly suppressed RUNX2 expression in these two cell lines. (b) Real-time PCR quantitatively measures RUNX2 mRNA $\left({ }^{*} P<0.05\right.$ versus control (FBS $\left.\left.0 \%\right)\right)$. (c) Immunocytofluorescence labeling of Runx2 shows induction of Runx2 expression after serum stimulation in WRO and TPC-1 cells. Cellular nuclei are labeled with DAPI (small square). (d) Western blotting. Serum stimulation increases Runx2 protein expression in WRO and TPC-1 cells, whereas U0126 reduces the expression of Runx2 in both cell lines. U0126 entirely blocks ERK phosphorylation in WRO cells and partially blocks those in TPC-1 cells. 
immunocytofluorescence and MAPK/ERK inhibition by western blotting (Figure 3d).

\section{Runx2 Association with EMT-Related Molecules and Angiogenic Factors}

To explore the roles of Runx2 in thyroid carcinoma, we performed siRNA-mediated gene silencing against Runx 2 . We selected WRO and 8305 cells based on their endogenous expression of Runx2. Conventional RT-PCR and real-time PCR confirmed effective knockdown of RUNX2 mRNA in WRO and $8305 \mathrm{C}$ (Figure $4 \mathrm{a}$ and b). As shown in Figure 4c, Runx2 protein was positive in the nuclei of control cells, whereas it was negative or weakly positive in the nuclei of RUNX2 siRNA-treated WRO or 8305C cells.

Following confirmation of the effective Runx 2 knockdown, we next examined the potential targets of Runx2, focusing on EMT-related molecules (SNAI1, SNAI2, SNAI3 and TWIST1), MMPs (MMP1, MMP2 and MMP9) and vasculogenic factors (VEGFA and VEGFC). Through RT-PCR, we found that Runx2 silencing resulted in a decreased mRNA expression of SNAI2 and VEGFC in WRO cells by (Figure 4a). The Runx2-silenced 8305C cells also showed downregulation of SNAI2, SNAI3, TWIST1, MMP2, VEGFA and $V E G F C$. The significant downregulation in these genes was quantitatively validated in both cell lines using real-time PCR $(P<0.05 ;$ Figure $4 \mathrm{~b})$. Immunocytofluorescence staining showed decreased expression of Snail2/Slug (SNAI2) and VEGFC in Runx2 siRNA/WRO cells compared with control cells (Figure 4c). We confirmed downexpression of MMP2 in Runx2 siRNA/8305C cells. Runx2 knockdown presented no alteration of SNAI1, MMP1 and MMP9 in either cell line. The results of PCR and immunocytofluorescence were further validated by western blotting (Figure $4 \mathrm{~d}$ ).

\section{Runx2 Silencing Impedes Invasion of Thyroid Carcinoma Cells}

Having determined that Runx2 targets EMT-related molecules and MMP in thyroid cancer cells, we next examined the contribution of Runx2 to cancer cell invasion using transwell assays. In WRO cells that express endogenous Runx2, we found that Runx2 silencing unequivocally suppressed cell invasion compared with the control $(P=0.03)$, whereas there was no significant effect on cell migration $(P=0.112$; Figure $5 \mathrm{a}$ and $\mathrm{b}$ ).

\section{DISCUSSION}

Transcription factor Runx2 (RUNX2/CBFA1) is a member of the Runx family, and has an essential role in osteoblastic differentiation and osteogenesis by regulating osteoblastspecific genes such as osteopontin and osteocalcin. ${ }^{19}$ Recently, its alternative role has been emphasized in carcinogenesis. ${ }^{20}$ In this study, we found there was focal and weak expression of Runx2 in normal thyroid and benign thyroid lesions, and diffuse and intense expression in thyroid carcinomas including PCs, FCs and UCs.
The function of thyroid follicles is heterogeneously synchronized in a normal thyroid under the same supply of thyrotropin in the blood. Our previous studies demonstrated this follicular heterogeneity in normal thyroid with enzyme histochemistry for ecto-5'-nucleotidase and with immunohistochemistry for thyroid-specific proteins including TTF-1, Tg, thyroid peroxidase and pendrin. ${ }^{17,21}$ Recently, Endo and Kobayashi ${ }^{22}$ reported that Runx $2^{+/-}$heterozygous mice are in a hypothyroid state. In this seminal study, they demonstrated that Runx2 binds to the Tg promoter region and regulates $\mathrm{Tg}$ expression in FRTL-5 cells. Our immunohistochemistry revealed focal expression of Runx2 in $29 \%$ of normal thyroids. We presume that heterogeneous expression of Runx2 immunoreactivity reflects functional heterogeneity of thyroid follicles. However, the physiological role of Runx2 in follicular cells of normal or non-neoplastic thyroid lesions is still to be elucidated.

We showed that Runx2 is upregulated in various types of follicular cell-derived thyroid carcinomas. This finding suggested to us that enhanced expression of Runx2 has some tumor-promoting properties in thyroid carcinomas. Indeed, a Runx2 role has been emphasized in several human cancers such as prostate cancer and breast cancer. ${ }^{20}$ Runx2 expression is higher in prostate cancer and invasive breast cancer compared with corresponding non-neoplastic or non-invasive lesions. ${ }^{23-25}$ Enhanced Runx2 expression in prostate cancer is associated with disease progression including higher Gleason scores. ${ }^{8}$ In breast cancers with a high labeling index of Runx2, the clinical outcome of the breast cancer was worse; Runx 2 is used as an independent prognostic factor in these patients. ${ }^{23}$

An increasing number of studies have suggested oncogenic functions of Runx 2 in carcinoma cells. It is reported that Runx2 regulates expression of survivin that inhibits caspase activity, leading to an antiapoptotic effect, and the report demonstrated that signaling by bone morphogenic protein (BMP) enhances Runx2 expression in prostate cancer. ${ }^{26}$ Zhang $\mathrm{et} \mathrm{al}^{27}$ reported that Runx2 binds the promoter region of LGAL3 (galectin-3) and regulates galectin-3 levels in the pituitary tumors. The relation of Runx 2 and galectin- 3 would be challenging issue for future studies in thyroid cancers, as malignant thyroid tumors frequently express higher level of galectin-3 as well as Runx2. ${ }^{28}$ It leads us to surmise that galectin-3, which plays oncogenic functions in human cancers and ancillary diagnostic marker of thyroid carcinomas, is also a direct target gene of Runx2.

Runx2 is also associated with invasion and metastasis of prostatic carcinoma cells by regulating MMP9, MMP13, VEGF and osteopontin. ${ }^{8,20}$ In breast carcinoma cells, expression of Runx2 is shown to be important in the positive regulation of angiogenic factors, and Runx2-regulated MMPs are functionally related to the invasion properties of breast cancer cells. ${ }^{29,30}$ Likewise, our current study demonstrated that Runx2 expression is involved with positive regulation of angiogenic factors (VEGFA, VEGFC), MMP2 and EMTrelated molecules (SNAI2, SNAI3, TWIST1) in thyroid 
a
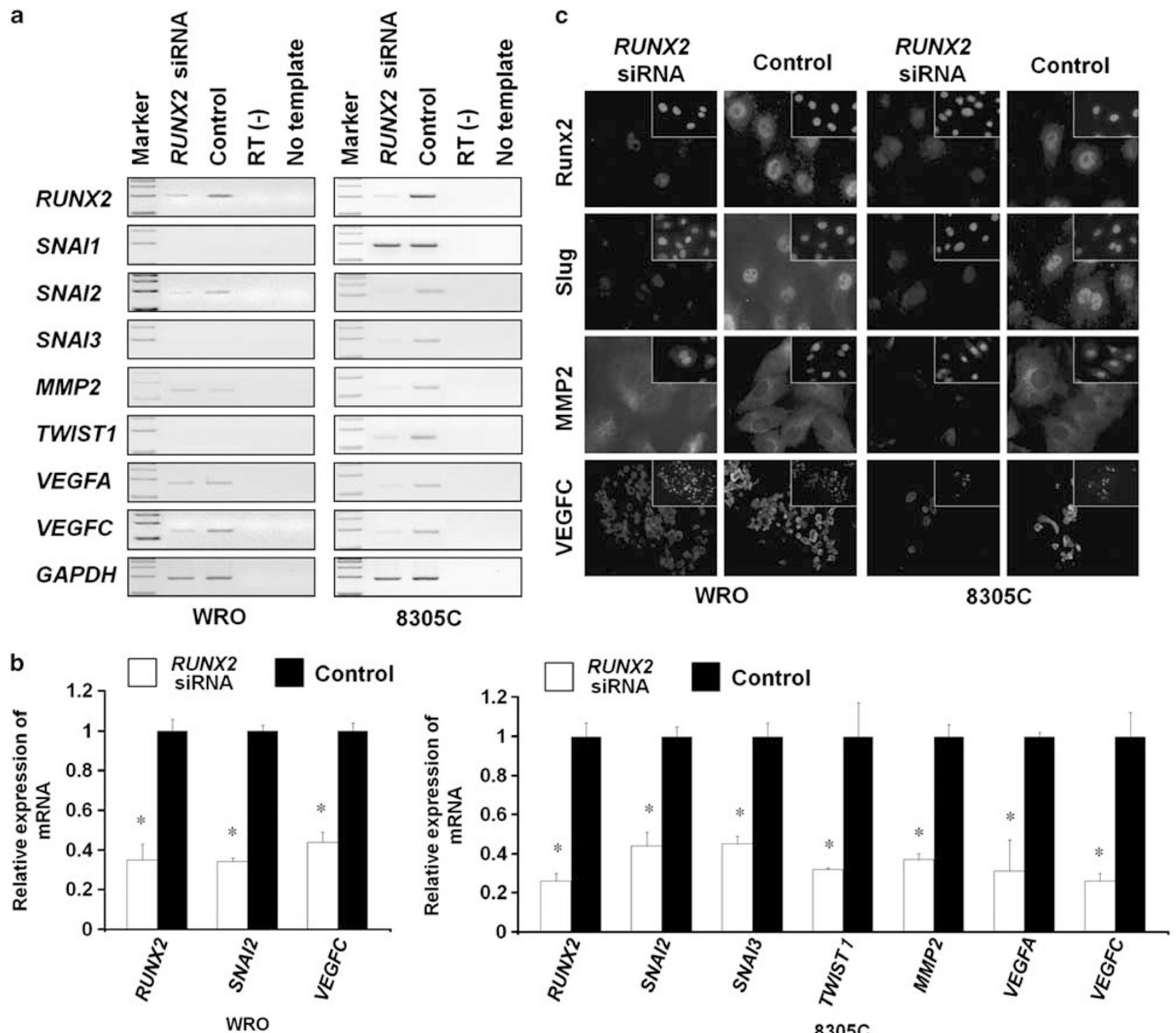

d

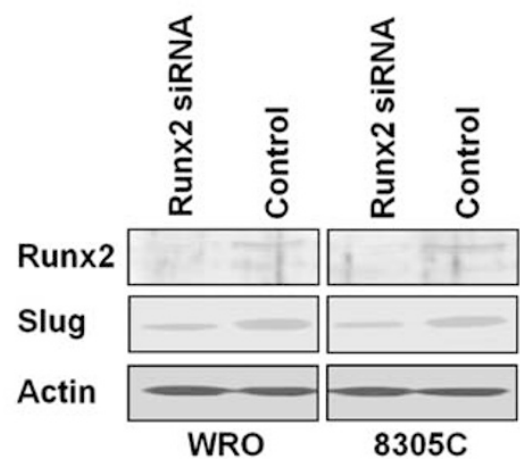

Figure 4 Runx2 associated with EMT-related molecules, MMP and vasculogenic factors. (a) RT-PCR. Runx2 silencing results in decreased mRNA expression of SNAI2 and VEGFC in WRO cells. Runx2-silenced 8305 C cells show downregulation of SNAI2, SNAI3, TWIST1, MMP2, VEGFA and VEGFC. (b) Real-time PCR quantitatively validates the significant downregulation of genes in both cell lines $(P<0.05)$. (c) Immunocytofluorescence shows decreased expression of Snail2/Slug (SNA/2) and VEGFC in Runx2 siRNA/WRO cells compared with control cells. Downexpression of MMP2 is confirmed in Runx2 siRNA/8305C cells. Cellular nuclei are labeled with DAPI (small square). (d) Western blotting demonstrates effective Runx2 knockdown and downregulation of Snail2/Slug (SNAI2) in Runx2 siRNA cells. 
b

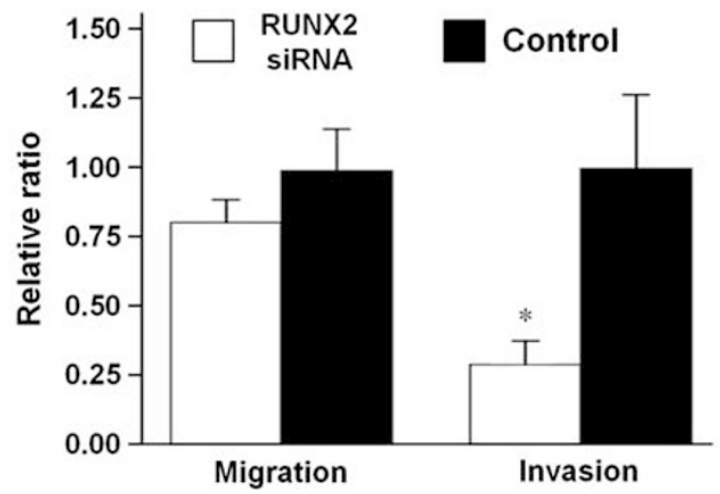

Figure 5 Runx2 silencing impedes thyroid carcinoma cell invasion. (a) Runx2 siRNA cells and control cells are allowed to pass through Matrigel-coated (for invasion) or uncoated (for migration) filters.

(b) Runx2 silencing significantly suppresses thyroid carcinoma cell invasion $\left({ }^{*} P=0.03\right)$, whereas there is no significant effect on cell migration.

carcinoma. Furthermore, we showed that Runx2 silencing repressed the invasion of thyroid carcinoma cells in the transwell cell invasion assay. These data suggest a key role of Runx2 in the molecular mechanism of thyroid cancer invasiveness.

The EMT phenomenon, which is characterized by loss of cell adhesion, repression of E-cadherin expression and increased cell mobility has been implicated in cancer invasiveness and metastasis. Vasko et $\mathrm{al}^{13}$ demonstrated that EMT-related molecules, including Runx2, were upregulated, particularly in the invasive region of the PC. They also revealed that vimentin, an overexpressed EMT marker in the PC, is required for thyroid cancer cell invasion. In our study, we observed a high frequency and high intensity of Runx2 immunopositivity in various types of invasive thyroid carcinomas. Furthermore, we showed Runx2 to be positively linked with the expression of Twist1 and the Snail family in thyroid carcinoma cells. Twistl is a helix-loop-helix transcription factor and a promoter of the EMT process mediated by E-cadherin suppression. The Snail family is a group of zinc-finger transcription factors consisting of Snail1/SNAI1, Snail2/Slug/SNAI2 and Snail3/SNAI3 ${ }^{31}$ This family of transcription factors also contributes to EMT by E-cadherin downregulation. Recently, Casas et $a l^{32}$ demonstrated Snail2/ Slug as the direct transcription target of Twist1, and it is required for the Twist1-induced E-cadherin suppression. Although expression of Twistl and the Snail transcription factors are regulated by an integrated and complex signaling pathway, our current data suggest that Runx2 is one of the regulators of these EMT-inducing factors. Taken together, inhibition of Runx 2 would be a potential therapeutic approach against invasion and metastasis of thyroid carcinomas.

In osteoblasts, MAPK/ERK activation enhances the transcriptional activity of Runx2 and promotes osteoblast differentiation, ${ }^{33,34}$ which supports the results of our study. Expression of Runx2 was significantly suppressed by selective MEK inhibition, suggesting that regulation of Runx2 is dependent on the MAPK/ERK pathway in thyroid carcinoma cells. Persistent activation of MAPK/ERK signaling because of genetic alteration, such as RET rearrangements, NTRK rearrangements and activating somatic mutations in RAS and $B R A F$ oncogenes, is a feature of most follicular cell-derived carcinomas including FCs, PCs and UCs. ${ }^{1}$ The enhanced expression of Runx2 observed in various types of thyroid carcinomas is probably attributable to intrinsic gene abnormalities resulting in aberrant activation of MAPK/ERK signaling. However, we should take notice that partial loss of serum effect against Runx2 by MEK inhibition also suggests the contribution of alternative pathways other than MAPK/ ERK signaling.

In this study, RUNX1 mRNA is upregulated in PCs compared with normal thyroid tissues along with the expression of RUNX2. In addition, the majority of follicular cell-derived thyroid carcinoma cell lines expressed RUNX1 and RUNX2 mRNA, whereas two of four PC cases had a lower expression of RUNX3 mRNA, and RUNX3 mRNA was detected in only one cell line. These divergent expression patterns of the Runx family suggest that Runx 1 and Runx 3 also might play a role in the development of thyroid carcinoma. However, further studies regarding Runx1 and Runx3 are needed to clarify their biological roles in the progression of thyroid carcinomas.

In conclusion, this study provides insight into a potential molecular mechanism of thyroid cancer invasion. Our data suggest that enhanced Runx2 is linked to tumor invasion and metastasis of thyroid carcinoma by regulating EMT-related molecules, MMP and angiogenic/lymphangiogenic factors.

\section{ACKNOWLEDGEMENT}

We thank Ms Miyuki Ito, Ms. Mikiko Yoda and Mr Yoshihito Koshimizu for technical support and Ms Kayoko Kono for executive assistance. 


\section{DISCLOSURE/CONFLICT OF INTEREST}

The authors declare no conflict of interest.

1. Kondo T, Ezzat S, Asa SL. Pathogenetic mechanisms in thyroid follicular-cell neoplasia. Nat Rev Cancer 2006;6:292-306.

2. Kondo T, Asa SL, Ezzat S. Epigenetic dysregulation in thyroid neoplasia. Endocrinol Metab Clin North Am 2008;37:389-400.

3. Komori T. Regulation of skeletal development by the Runx family of transcription factors. J Cell Biochem 2005;95:445-453.

4. Maki K, Yamagata T, Mitani K. Role of the RUNX1-EVI1 fusion gene in leukemogenesis. Cancer Sci 2008;99:1878-1883.

5. Kayed H, Jiang $X$, Keleg $S$, et al. Regulation and functional role of the Runt-related transcription factor-2 in pancreatic cancer. $\mathrm{Br} \mathrm{J}$ Cancer 2007;97:1106-1115.

6. Pratap J, Lian JB, Javed A, et al. Regulatory roles of Runx2 in metastatic tumor and cancer cell interactions with bone. Cancer Metastasis Rev 2006;25:589-600.

7. Lee YM. Control of RUNX3 by histone methyltransferases. J Cell Biochem 2011;112:394-400.

8. Akech J, Wixted JJ, Bedard K, et al. Runx2 association with progression of prostate cancer in patients: mechanisms mediating bone osteolysis and osteoblastic metastatic lesions. Oncogene 2010;29:811-821.

9. Sun X, Wei L, Chen Q, et al. HDAC4 represses vascular endothelial growth factor expression in chondrosarcoma by modulating RUNX2 activity. J Biol Chem 2009;284:21881-21890.

10. Papachristou DJ, Papachristou GI, Papaefthimiou OA, et al. The MAPKAP-1/-Runx2 signalling axes are implicated in chondrosarcoma pathobiology either independently or via up-regulation of VEGF. Histopathology 2005;47:565-574.

11. Baniwal SK, Khalid $O$, Gabet $Y$, et al. Runx2 transcriptome of prostate cancer cells: insights into invasiveness and bone metastasis. Mol Cancer 2010;9:258.

12. Yuen HF, Kwok WK, Chan KK, et al. TWIST modulates prostate cancer cell-mediated bone cell activity and is upregulated by osteogenic induction. Carcinogenesis 2008;29:1509-1518.

13. Vasko V, Espinosa AV, Scouten W, et al. Gene expression and functional evidence of epithelial-to-mesenchymal transition in papillary thyroid carcinoma invasion. Proc Natl Acad Sci USA 2007;104:2803-2808.

14. DeLellis RA, Lloyd RV, Heitz PU, et al. World Health Organization Classification of Tumours. Pathology and Genetics of Tumours of Endocrine Organs. IARC Press: Lyon, France, 2004.

15. Kondo T, Nakazawa T, Murata S, et al. Enhanced B-Raf protein expression is independent of $\mathrm{V} 600 \mathrm{E}$ mutant status in thyroid carcinomas. Hum Pathol 2007;38:1810-1818.

16. Katoh R, Miyagi E, Nakamura N, et al. Expression of thyroid transcription factor-1 (TTF-1) in human $C$ cells and medullary thyroid carcinomas. Hum Pathol 2000;31:386-393.
17. Kondo T, Nakamura N, Suzuki K, et al. Expression of human pendrin in diseased thyroids. J Histochem Cytochem 2003;51:167-173.

18. Ma DF, Kondo T, Nakazawa $T$, et al. Hypoxia-inducible adenosine A2B receptor modulates proliferation of colon carcinoma cells. Hum Pathol 2010;41:1550-1557.

19. Chiou WF, Lee $\mathrm{CH}$, Liao JF, et al. 8-Prenylkaempferol accelerates osteoblast maturation through bone morphogenetic protein-2/p38 pathway to activate Runx2 transcription. Life Sci 2011;88:335-342.

20. Blyth K, Vaillant F, Jenkins A, et al. Runx2 in normal tissues and cancer cells: a developing story. Blood Cells Mol Dis 2010;45:117-123.

21. Kondo T, Nakazawa T, Murata SI, et al. Expression of CD73 and its ecto5 -nucleotidase activity are elevated in papillary thyroid carcinomas. Histopathology 2006;48:612-614.

22. Endo T, Kobayashi T. Runx2 deficiency in mice causes decreased thyroglobulin expression and hypothyroidism. Mol Endocrinol 2010;24:1267-1273.

23. Onodera Y, Miki Y, Suzuki T, et al. Runx2 in human breast carcinoma: its potential roles in cancer progression. Cancer Sci 2010;101:2670-2675.

24. Chua CW, Chiu YT, Yuen HF, et al. Suppression of androgenindependent prostate cancer cell aggressiveness by FTY720: validating Runx2 as a potential antimetastatic drug screening platform. Clin Cancer Res 2009;15:4322-4335.

25. Nagaraja GM, Othman M, Fox BP, et al. Gene expression signatures and biomarkers of noninvasive and invasive breast cancer cells: comprehensive profiles by representational difference analysis, microarrays and proteomics. Oncogene 2006;25:2328-2338.

26. Lim $M$, Zhong $C$, Yang $S$, et al. Runx2 regulates survivin expression in prostate cancer cells. Lab Invest 2010;90:222-233.

27. Zhang $\mathrm{H}-\mathrm{Y}$, Jin L, Stilling GA, et al. RUNX1 and RUNX2 upregulate Galectin3 expression in human pituitary tumors. Endocrine 2009;35:101-111.

28. Fischer S, Asa SL. Application of immunohistochemistry to thyroid neoplasms. Arch Pathol Lab Med 2008;132:359-372.

29. Pratap J, Javed A, Languino LR, et al. The Runx2 osteogenic transcription factor regulates matrix metalloproteinase 9 in bone metastatic cancer cells and controls cell invasion. Mol Cell Biol 2005;25:8581-8591.

30. Javed A, Barnes GL, Pratap J, et al. Impaired intranuclear trafficking of Runx2 (AML3/CBFA1) transcription factors in breast cancer cells inhibits osteolysis in vivo. Proc Natl Acad Sci USA 2005;102:1454-1459.

31. Wu Y, Zhou BP. Snail: more than EMT. Cell Adh Migr 2010;4:199-203.

32. Casas E, Kim J, Bendesky A, et al. Snail2 is an essential mediator of Twist1-induced epithelial mesenchymal transition and metastasis. Cancer Res 2011;71:245-254.

33. Costessi A, Pines A, D'Andrea $P$, et al. Extracellular nucleotides activate Runx2 in the osteoblast-like HOBIT cell line: a possible molecular link between mechanical stress and osteoblasts' response. Bone 2005;36:418-432.

34. Franceschi RT, Xiao G. Regulation of the osteoblast-specific transcription factor, Runx2: responsiveness to multiple signal transduction pathways. J Cell Biochem 2003;88:446-454. 